\title{
Methods for calculating the average particulate matter concentrations in the Krasnoyarsk city ground layer
}

\author{
Maxim I. Malimonov ${ }^{1}$, Alexander A. Pushkarev² and Oxana V. Sokolova ${ }^{1}$ \\ ${ }^{1}$ Federal Research Center Krasnoyarsk Science Center SB RAS, Krasnoyarsk, Russia \\ ${ }^{2}$ Institute of Computational Modelling SB RAS, Krasnoyarsk, Russia
}

\begin{abstract}
The paper considers a method based on the Voronoi diagram for calculating the average particulate matter concentration in the surface layer of the Krasnoyarsk city air environment. We have used two methods to achieve this goal. The first method relied on buffer zones built in the immediate vicinity around monitoring posts. The second one uses the boundaries of the city of Krasnoyarsk.
\end{abstract}

\section{Keywords}

JavaScript, JSON, air pollution, PM2.5.

\section{Introduction}

Analysis of the results of monitoring the state of atmospheric air, determining the level of air pollution in the city as a whole is the subject of numerous discussions. At present, the level of atmospheric pollution at time intervals from 20 minutes to twenty-four hours researchers estimate by the value of the maximum value of the parameter recorded for the selected interval. This approach does not evaluate the average concentration of the measured impurity for the city as a whole. The paper considers a method for calculating the average concentration of suspended particles in the surface layer of the Krasnoyarsk air environment based on the Voronoi diagram. The average value is calculated as follows. According to the original point data, a Voronoi diagram is constructed. After that, boundary conditions are imposed on the diagram, which is used as the contour of the city territory. Next, the weighted average is calculated, where the weight is the area of the Voronoi polygons. The application of such a calculation for the analysis of data from the air monitoring system of the Krasnoyarsk Scientific Center of the SB RAS is demonstrated.

\subsection{Data processing}

This paper discusses an approach for calculating the average concentration of suspended particles using the Voronoi diagram. The data for the work were taken from the environmental monitoring network of the Federal Research Center of the KSC SB RAS. There is software and hardware for collecting and storing data to store all the information [1].

SDM-2021: All-Russian conference, August 24-27, 2021, Novosibirsk, Russia

冈malimonov.mi@ksc.krasn.ru (M. I. Malimonov); pushkarev@icm.krasn.ru (A. A. Pushkarev)

(c) (1) ๑ 2021 Copyright for this paper by its authors. Use permitted under Creative Commons License Attribution 4.0 International (CC BY 4.0).

CEUR Workshop Proceedings (CEUR-WS.org) 
The average daily data for the whole month is taken for the analysis. Visualization subsystem environmental data based on Javascript libraries is used to display the data [2]. The dline.js library is responsible for displaying polygons and contours [3].

\section{Results and Discussion}

The first step for analyzing the data is to select the period with the most noticeable contamination. These periods are February 2019, November 2020 and from the end of December 2020 to the beginning of January 2021. The unfavorable meteorological conditions (UMC) regime was announced from 19:00 February 8, 2019, to 19:00 February 13, 2019 [4]. Next time the UMC was announced from November 27 to November 30 . In the third selected period, the UMC was announced from December 26, 2020, to January 2, 2021.

The average value is calculated as follows. Interpolation is built using Voronoi diagrams based on the initial point data, and this makes it easy to divide the city into some areas of influence of each monitoring station. Voronoi diagrams are built using the turf.js library, which provides many different functions for processing spatial data in JavaScript

After building Voronoi diagrams, the layer is cropped along the required contour. In this work, two variants of the competition were used: the first one is along the buffer zones around each monitoring station with a radius of $3 \mathrm{~km}$; the second one is along the administrative boundaries of the city of Krasnoyarsk. The buffer zone is the area of influence of one monitoring post. Competing is carried out using the npm package polygon-clipping, which allows one to perform operations to trim polygons, merge polygons or subtract the boundaries of one polygon from another.

The next step is to calculate the area of each resulting polygon, which is also carried out using the truf.js library. The result is a GeoJSON file containing a set of polygons, the area polygon, and the value of the point around which each Voronoi polygon was built.

Next, the average pollution value for the entire city is calculated. The calculation is made according to the arithmetic weighted average formula (1), where the weight is the area of each Voronoi polygon.

$$
\bar{x}=\frac{\sum_{i=1}^{n} w_{i} \cdot x_{i}}{\sum_{i=1}^{n} w_{i}},
$$

where $w_{i}$ is the area of each polygon obtained as a result of constructing Voronoi diagrams, $x_{i}$ is the value of a point that hits this polygon.

The second approach uses not buffer zones but the boundaries of the city of Krasnoyarsk. Figure 1 shows the result of building buffer zones around monitoring posts. This figure shows the peak of pollution on February 12, 2019.

Figure 2 shows the construction of a Voronoi diagram within the boundaries of the city of Krasnoyarsk for the same period. Monitoring posts outside the city boundaries are taken into account when constructing a pollution map.

Figure 3 displays the comparison of mean concentrations of suspended particles Card PM2.5 in February 2019, obtained by using different approaches. 


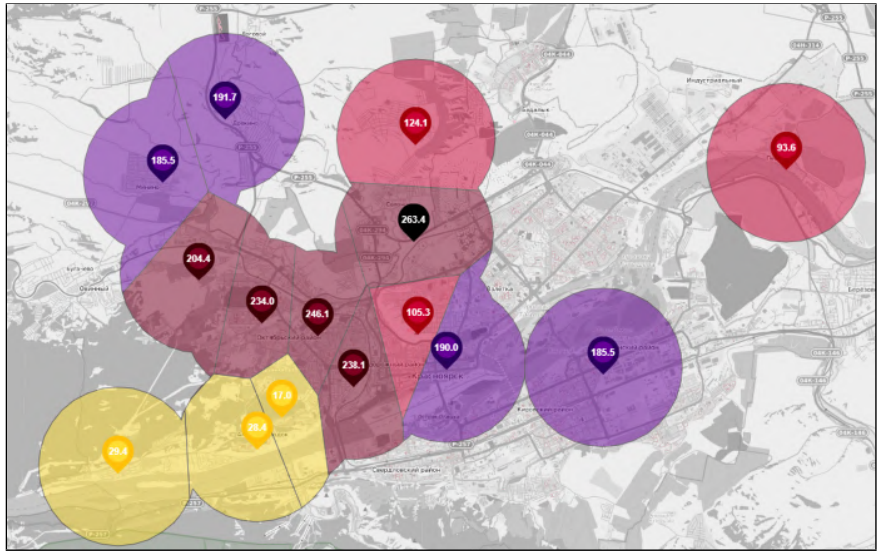

Figure 1: Buffer zones separated by a Voronoi diagram.

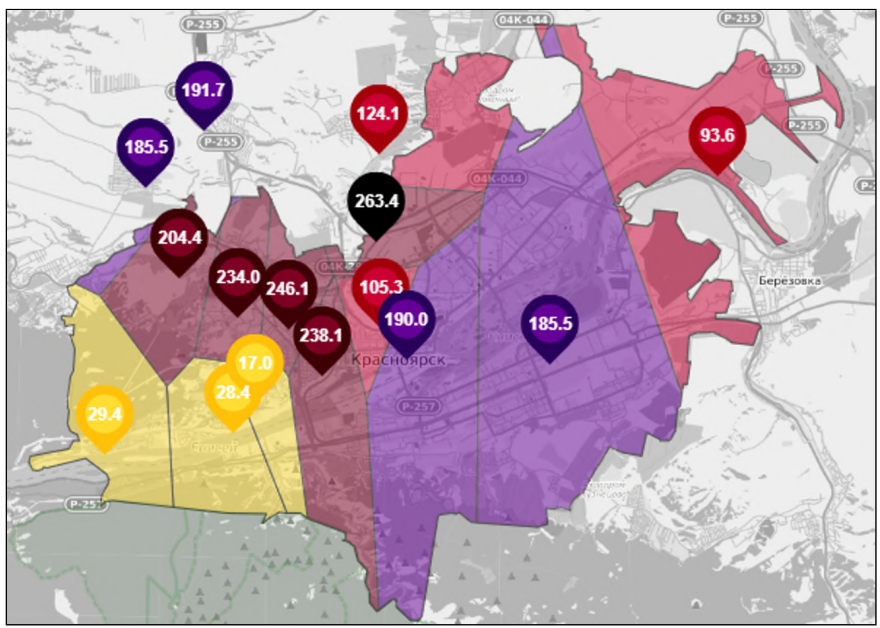

Figure 2: The city of Krasnoyarsk, divided by the Voronoi diagram.

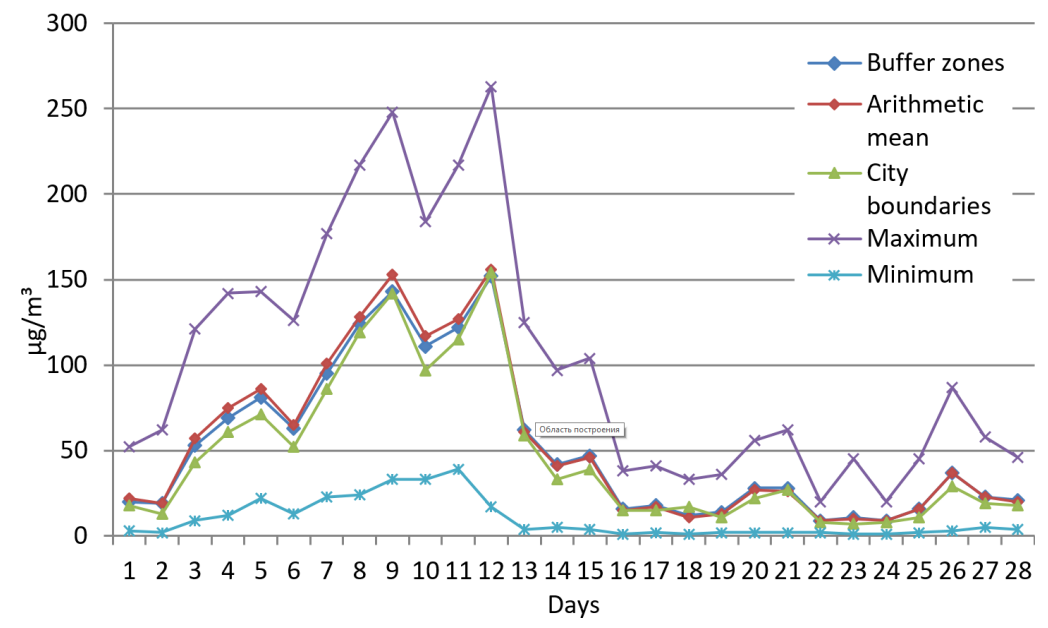

Figure 3: Comparison of average concentrations in February 2019. 
The most significant differences begin at high concentrations. If we consider the average concentrations together with the maximum and minimum concentrations, then some differences say that the city's pollution is highly uneven. One can also replace that averages and highs show the same trend, as opposed to lows.

Fifteen posts in the city of Krasnoyarsk were used for the previous calculations, located in different districts of Krasnoyarsk, and their number is sufficient to cover most of the city. In some areas, there are several monitoring posts at once. For the following calculations, some posts were removed from the districts of the city. Figure 4 shows the result of this construction.

In this case, other results were obtained, which show that this number of posts is enough to calculate average concentrations and display a trend, but in this case, some monitoring posts pull over a large share of pollution. In Figures 1 and 2, there were yellow zones in the Akademgorodok microdistrict, located in the Oktyabrsky district. We removed posts from this microdistrict and set up one monitoring post located in the Nikolaevka microdistrict in the

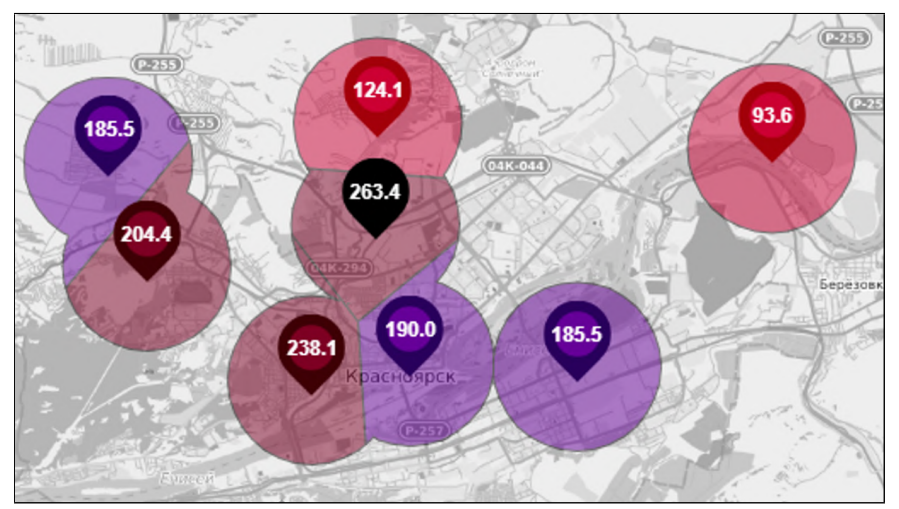

Figure 4: Buffer zones built with the exclusion of some posts and separated by a Voronoi diagram.

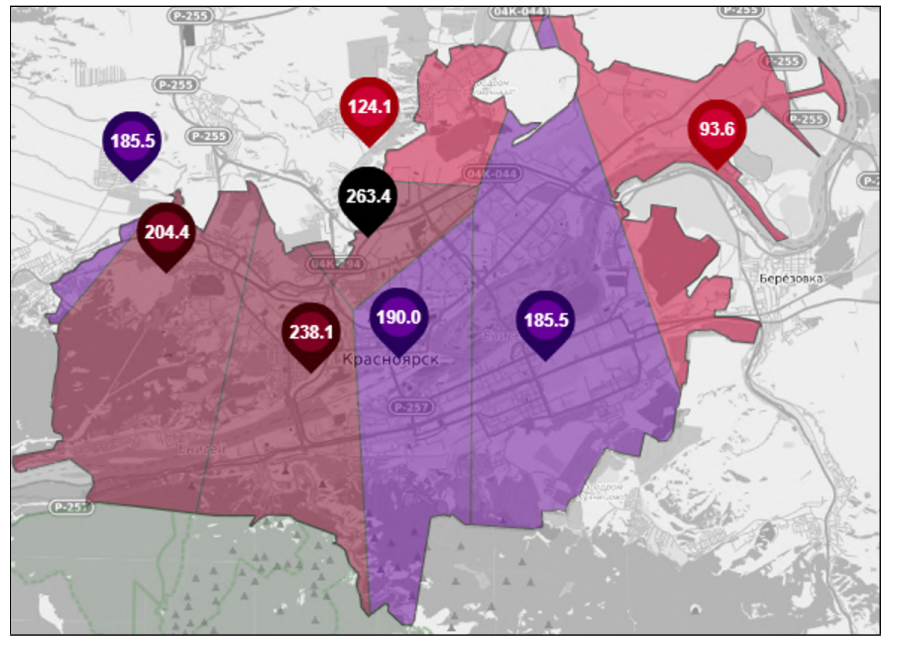

Figure 5: Krasnoyarsk city borders, separated by Voronoi diagram, except for some posts. 
Oktyabrsky district. The minimum values shifted quite strongly towards the average values, and the differences between the average values of the different approaches became smaller but increased in value. Figure 6 shows the calculation results without taking into account several monitoring posts.

These results show that the placement of one post per district is not enough. The city has a rather tricky terrain, and monitoring posts located in neighboring micro-districts can show completely different values, which differ tenfold.

Figure 7 shows the results of November 2020. The data below is calculated using all monitoring posts. Tables 1 and 2 show the results of comparing the average values obtained by different methods.

Based on the results shown in the tables, we can conclude that the difference between the "Arithmetic mean" and "Buffer zones" is not significant. The difference between Borders and Arithmetic mean is more tangible.

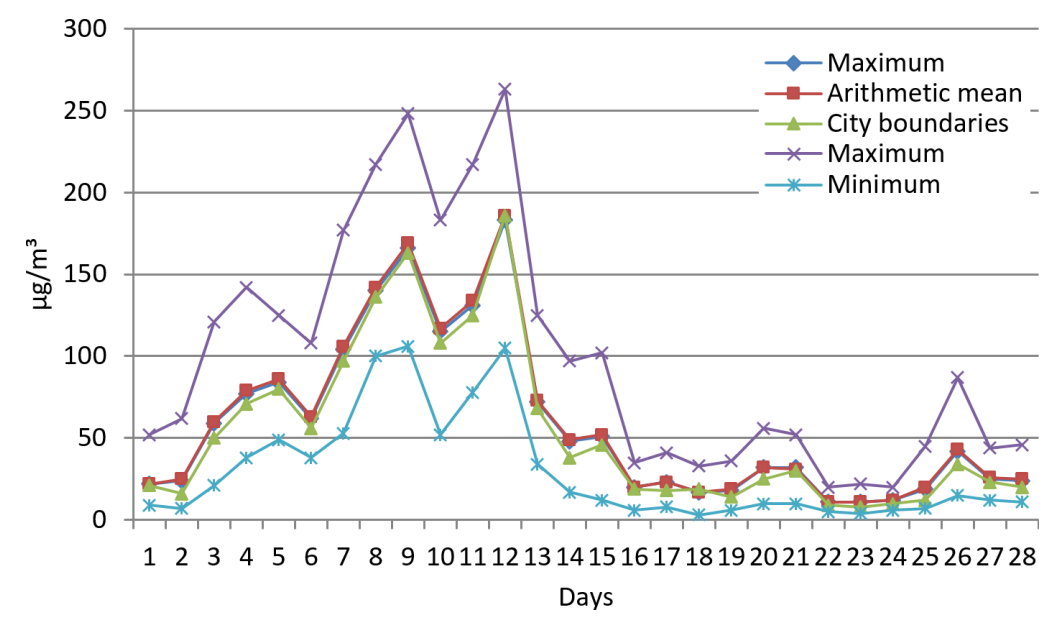

Figure 6: Comparison of average concentrations in February $2019\left(\mu \mathrm{g} / \mathrm{m}^{3}\right)$ excluding some monitoring stations.

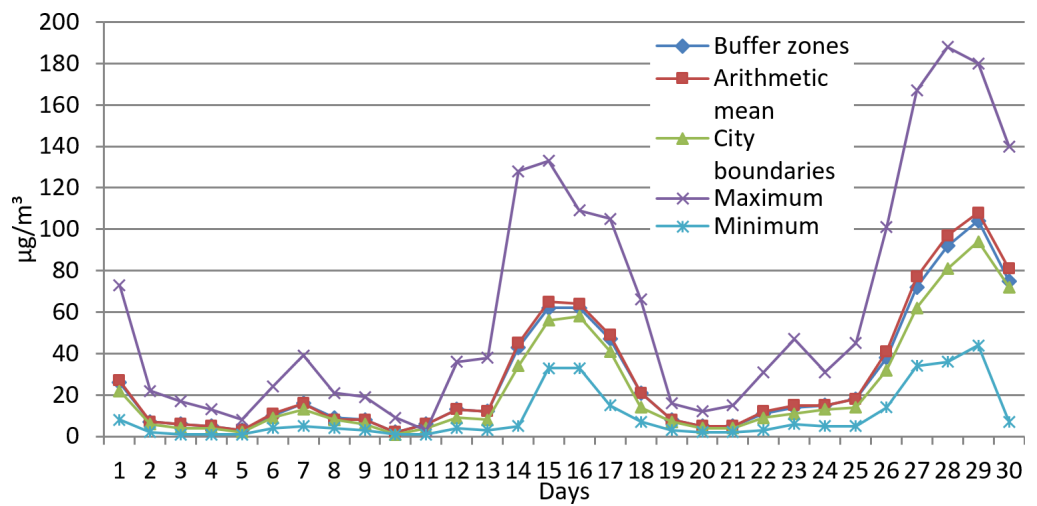

Figure 7: Comparison of average concentrations in November 2020 taking into account all monitoring stations. 
Table 1

Two-sample $t$-test for November 2020. Comparison of "Arithmetic mean" and "Buffer zones" values.

\begin{tabular}{lrr}
\hline & Arithmetic mean & Buffer zones \\
\hline Average & 28.33333333 & 27.16666667 \\
Variance & 934.0229885 & 834.7643678 \\
Observations & 30 & 30 \\
Combined variance & 884.3936782 & \\
Hypothetical difference of averages & 0 & \\
df & 58 & \\
$t$-statistics & 0.151939119 & \\
$P(T \leq t)$ one-sided & 0.439881121 & \\
$t$ critical one-sided & 1.671552762 & \\
$P(T \leq t)$ two-sided & 0.879762243 & \\
$t$ critical two-sided & 2.001717484 & \\
\hline
\end{tabular}

\section{Table 2}

Two-sample $t$-test for November 2020. Comparison of "Arithmetic mean" and "Boundaries" values.

\begin{tabular}{lrr}
\hline & Arithmetic mean & Buffer zones \\
\hline Average & 28.333333333 & 23.4 \\
Variance & 934.0229885 & 699.0068966 \\
Observations & 30 & 30 \\
Combined variance & 816.5149425 & \\
Hypothetical difference of averages & 0 & \\
df & 58 & \\
$t$-statistics & 0.668657964 & \\
$P(T \leq t)$ one-sided & 0.25318317 & \\
$t$ critical one-sided & 1.671552762 & \\
$P(T \leq t)$ two-sided & 0.50636634 & \\
$t$ critical two-sided & 2.001717484 & \\
\hline
\end{tabular}

Figure 8 shows the results of the calculation, in which several monitoring posts were also removed. It can be seen that the minimum values have not changed much compared to Figure 7. That is because the post in Akademgorodok showed high values, and even with its exclusion, there were no significant changes. The values at this post are dependent on the wind. If the wind is blowing to the east, this post shows shallow values compared to the rest. During the UMC in November, the wind blew mainly to the north and at a low speed.

In the examples above, it is noticeable that the maximum values are not changed as when considering all the posts as without taking into account several monitoring posts. This is due to the fact that the maximum values are usually shown by the posts located in Solontsy or the city center. The posts in the city center show different results, but not significantly different from each other. Even if we take one monitoring post from the center, it will not change the overall picture. There is only one monitoring post in Solontsy, and it was not ruled out.

Figure 9 shows the results for the third selected period. In this case, the UMC affects two 


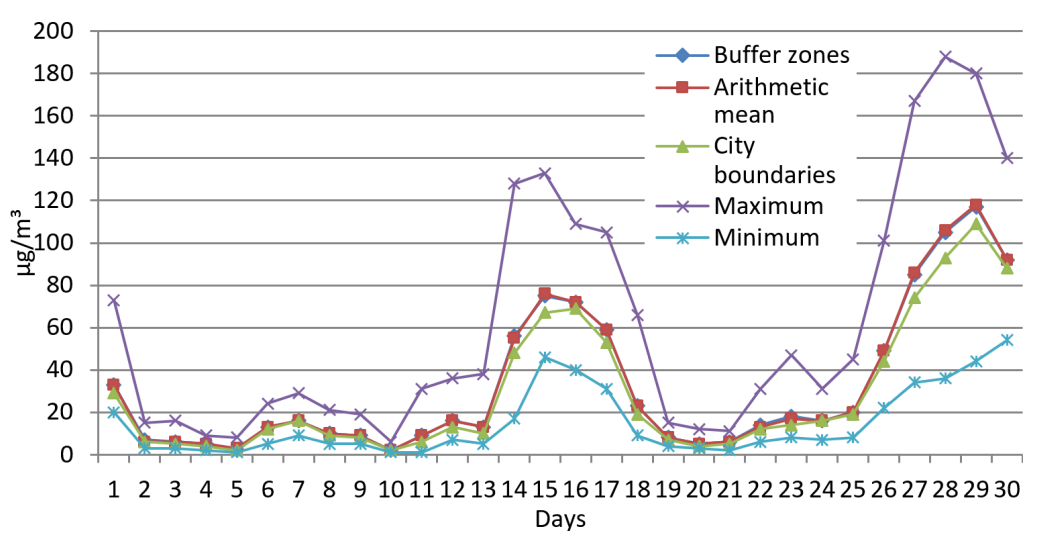

Figure 8: Comparison of average concentrations for November 2020 excluding several monitoring stations.

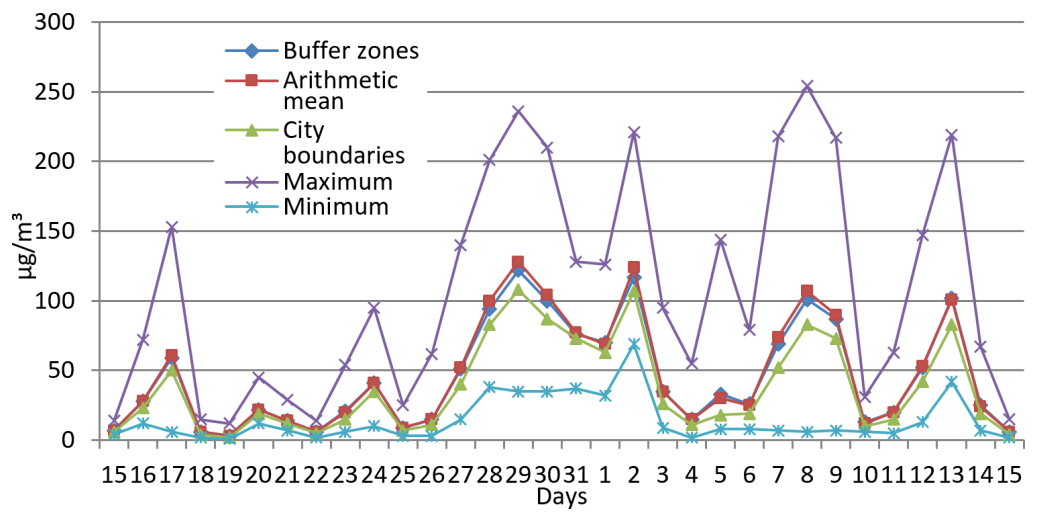

Figure 9: Comparison of average concentrations for November 2020 excluding several monitoring stations.

months at once, and we decided to take a thorough month, starting from December 15 to January 15.

Figure 9 shows that the minimum values correlate with the average values only on the days of the UMC regime. On other days, the wind was more than $3 \mathrm{~m} / \mathrm{s}$, and Akademgorodok shows the minimum values that are cut off from the city's values.

\section{Conclusions}

These results lead to several conclusions. 15-16 monitoring posts are enough to calculate average concentrations in the city, but one can lose the detail of the spread of pollution if reducing their number. The cases considered show that the wind has a relatively strong effect on the spread of pollution. The rugged terrain of the city requires the installation of many monitoring posts to display a high-quality picture. Even the exclusion of neighboring monitoring posts significantly changes the minimum values. Different methods for calculating average concentrations do not differ dramatically in values. 
The subsequent work will explore how to place monitoring posts in the city to reflect a quality picture and how many monitoring posts are needed to achieve this goal.

\section{References}

[1] A. Tokarev, Software and technology for collecting and storage serial observations of the environment, InterCarto. InterGIS 20 (2014) 241-249. doi:10.24057/ 2414-9179-2014-1-20-241-249.

[2] M. Malimonov, Software tools for interactive visualization of environmental data, in: Open Conference of Young Scientists of the ICM SB RAS on Mathematical Modeling and Information Technologies, ICM SB RAS, Krasnoyarsk, 2019, pp. 30-32.

[3] A. Pushkarev, Formation of isolines on a regular data in the web environment, in: Open Conference of Young Scientists of the ICM SB RAS on Mathematical Modeling and Information Technologies, ICM SB RAS, Krasnoyarsk, 2019, pp. 44-46.

[4] O. Yakubailik, V. Zavoruev, M. Malimonov, A. Pushkarev, Spatial analysis of air pollution in Krasnoyarsk, in: 2019 All-Russian Conference "Spatial Data Processing for Monitoring of Natural and Anthropogenic Processes", SDM 2019, Berdsk, CEUR Workshop Proceedings, volume 2534, 2020, pp. 491-496. 